\title{
Research Square \\ Gravitational Perturbations Correlated With The Asteroid Kinetic Impact Deflection Technique
}

\section{B.S Chagas ( $\square$ bruno.chagas@unesp.br)}

São Paulo State University (UNESP)
A. F. B. A. Prado
São Paulo State University (UNESP)
0. C. Winter
São Paulo State University (UNESP)

\section{Research Article}

Keywords:

Posted Date: February 2nd, 2022

DOI: https://doi.org/10.21203/rs.3.rs-1269348/v1

License: (1) This work is licensed under a Creative Commons Attribution 4.0 International License. Read Full License 


\title{
Gravitational perturbations correlated with the asteroid kinetic impact deflection technique.
}

\author{
Chagas,B.S $^{1, *}$, A. F. B. A. Prado ${ }^{1,2}$, and O. C. Winter ${ }^{1}$ \\ ${ }^{1}$ São Paulo State University (UNESP), Grupo de Dinâmica Orbital e Planetologia, Guaratinguetá-SP, Brazil. \\ ${ }^{2}$ Professor, Academy Engineering, RUDN University Miklukho-Maklaya Street 6, 117198, Moscow, Russia. , INPE: \\ National Institute for Space Research/São José dos Campos - SP - Brazil. \\ *bruno.chagas@unesp.br
}

\begin{abstract}
We will present the use of the kinetic impact technique as a way to deflect asteroids that may present some risk of collision with Earth at any given time. Within the work to be developed here, we intend to evaluate in more detail the possibility to deflect the orbit of the asteroid 101955 Bennu, applying variations in its velocity $(\Delta v)$ at different positions within its orbital period and measuring close encounters with planet Earth. We will see that, in a relatively long period of time, the asteroid has several close encounters with the planet, thus suffering a natural gravitational perturbation. With the application of the impulses, the relative distances change, causing variations in the energy of the asteroid and a large variation in the relative distance between the asteroid and Earth over a long period after the impulse. We present results in relation to the intensity of the applied impulse, which is important due to the size of the impactor to be considered and, for that, we mapped the positions of the impulses along a period of the orbit of the asteroid (M.I.O.A.). We finish by explaining what happens to the orbit of the asteroid during the periods of gravitational perturbation, since it undergoes to several "Swing Bys' that intensify the variations of the relative distances between the bodies after the impulses.
\end{abstract}

\section{Introduction}

Asteroids are small rocky or iron bodies that are remnants of the formation of the solar system ${ }^{1}$. These bodies can present great danger to life due to possible impacts with our planet. In addition, they can be sources of commercial and space exploration and also of scientific research and this problem has already been discussed in a range of publications ${ }^{2}$. We also run into the condition that these objects are generally very small in relation to planets and moons and, due to this factor, they have a weak gravitational attraction that causes their irregular shape ${ }^{3}$.

As a result, over the years, several proposals have come up with different techniques to cause perturbation in the orbit of the asteroid and thus deflect it. Methods such as causing the asteroid to vary in velocity through an impulse were tested, with an object causing an impact on the body, as well as the use of nuclear resources, solar sails, solar radiation collectors and laser systems ${ }^{4}$. Ledkov et al. ${ }^{5}$ showed that the use of gravitational maneuvers of a small asteroid with the Earth can be used in the planetary defense scenario. In this work they propose that, through small impulses in the orbit of a small asteroid along the approaches to Earth, a larger asteroid can be redirected to impact with a potentially dangerous asteroid.

Ahrens and Harris ${ }^{6}$ developed a work relating the forms of deflection of the asteroid with the fragmentation generated by them. They showed that, for perturbations that could take decades, velocity variations of at most $1 \mathrm{~m} / \mathrm{s}$ would be enough to generate significant changes in the orbit of the asteroid. With this work they showed that asteroids close to Earth can be deflected by varying their velocity, both increasing or decreasing, in relation to the Sun. However, they warn that for situations involving the need to deflect the body in a short period, some years, the variation should be hundreds of meters per second. In contrast, when an impulse is applied and the energy exerted on the asteroid exceeds a limit threshold, a catastrophic fragmentation is likely to occur, where it can result in damage to planet $\operatorname{Earth}^{7-9}$.

The kinetic impact deflection technique is gaining more and more priority in the scientific community, as it proves to be the most practical, resulting in the first real asteroid deflect mission with the DART mission, whose main objective is to quantify the deflection capacity of an asteroid using this technique ${ }^{10}$. The satellite called "Didymoon", will be impacted by a spacecraft, causing its orbit to be disturbed and, consequently, the orbit of the main body Didymos. The mission has support of the AIDA mission that will monitor the orbital change ${ }^{11-13}$.

Park and Ross ${ }^{14}$ showed that the change generated by an impulse is strongly dependent on the location of the impulse in the orbit, as well as the direction of the impulse in relation to the orbital velocity. In this work, they also argued that the impulse applied at the perihelion is the best for alert times longer than an orbital period.

Conway ${ }^{15}$ showed that, if an impactor is launched less than a year in advance of a possible collision of an asteroid with 
the Earth, the deflection obtained will be on the order of the diameter of the Earth for each $1 \mathrm{~m} / \mathrm{s}$ velocity change applied to the asteroid, but points out that, considering the mass of large asteroids, it would be very difficult to change its velocity by 1 $\mathrm{m} / \mathrm{s}$, thus warning that to be able to effectively apply the kinetic impact on moderate sized asteroids it is necessary to apply the impulse several years before a possible collision.

Izzo $^{16,17}$ presents important results in the segment of optimization of an analytical expression, where it is possible to show that the use of the kinetic impact technique ends up being simpler in relation to another impulsive method, showing the effectiveness of the technique.

The objective of this work is to evaluate the kinetic impact technique to deflect the orbit of potentially dangerous asteroids in relation to Earth considering the perturbations of the planets in the solar system and also how this relationship is made over a period of approximately 100 years after the impulses applied to the asteroids. So, we will vary the velocity of the object along its orbital period. Just like Negri et al. ${ }^{18}$. The asteroid chosen was the asteroid 101955 Bennu, which is part of the group of asteroids that have a near-Earth orbit (NEAs). This asteroid was a recent target of the Osiris Rex mission of NASA, gaining even more visibility within the scientific community.

\section{Methods}

In this work, we will analyze the gravitational effects involved in the kinetic impact asteroid deflection technique over a relatively long period. In this process we will solve the N-body problem using the Mercury integrator package ${ }^{19}$, considering the Burlish-Stoer integrator. We are considering all the planets in the solar system, the Moon and the target asteroid for our study, with the Sun as the central body.

The equation of motion for the N-body system with a body of mass much larger than the others can be written as follows ${ }^{20}$

$$
\begin{aligned}
\frac{d^{2}\left(\vec{r}_{i}\right)}{d t^{2}}+G\left(m_{n}+m_{i}\right) \frac{\vec{r}_{i}}{r_{i n}^{3}}= \\
G \sum_{j=1, j \neq i}^{N-1} m_{j} \quad\left(\frac{\vec{r}_{j}-\vec{r}_{i}}{r_{i j}^{3}}-\frac{\vec{r}_{j}}{r_{j n}^{3}}\right)
\end{aligned}
$$

where $\vec{r}_{i}$ and $\vec{r}_{j}$ are the position vectors of particles i and $\mathrm{j}$ in relation to the central body, $\mathrm{G}$ is the gravitational constant, $m_{n}$ is the mass of the central body, $m_{i}$ and $m_{j}$ are the masses of particles, $r_{i n}$ and $r_{j n}$ are the distances between particles $\mathrm{i}$ and $\mathrm{j}$ in relation to the central body and $r_{i j}$ is the relative distance between particles. The bodies used in the simulation will be presented later.

The equation below gives the two-body energy of asteroid (E) in relation to the semi-major axis. This equation is important for us, as we can get an idea of what physically happens to the asteroid over the period during which it is undergoing gravitational perturbation.

$$
E=-\frac{\mu}{2 a}
$$

where $\mu_{M 2}=G m_{M 2}$ and ' $\mathrm{a}$ ' is the semi-major axis of the asteroid.

With the evolution of the research, we realized that our results suffered a major contribution of maneuvers assisted by gravity, 'swing by', where this proved to be the main cause of alterations in the orbit of the asteroid. 'Swing by' maneuvers have been known for a long time in the astronomical scene and therefore are not new. The maneuver model (Figure 1) can be found in Prado $^{21}$ and Ferreira et al. ${ }^{22}$.

We can formulate the maneuver mathematically by

$$
\Delta E=-2 V_{2} V_{\infty} \sin (\delta) \sin (\psi)
$$

where $\Delta \mathrm{E}$ is the variation of the energy of the smaller body (The asteroid in our situation), $V_{2}$ is the velocity of $M_{2}$ in relation to $M_{1}, V_{\infty}$ is the particle velocity modulus at infinity before or after passing close to a body of mass $\mathrm{m}, \delta$ is the deflection angle and represents half the rotation of the velocity vector due to the 'swing by' and $\psi$ is the angle between the periapsis line and the line $M_{1}-M_{2}$, where,

$$
\sin (\boldsymbol{\delta})=\frac{1}{1+\frac{r_{p} V_{\infty}^{2}}{\mu_{2}}}
$$

since $r_{p}$ is the maximum approximation distance between M2, M3 and the body of mass $\mathrm{m}, \mu_{M 2}=G m_{M 2}{ }^{23}$. 


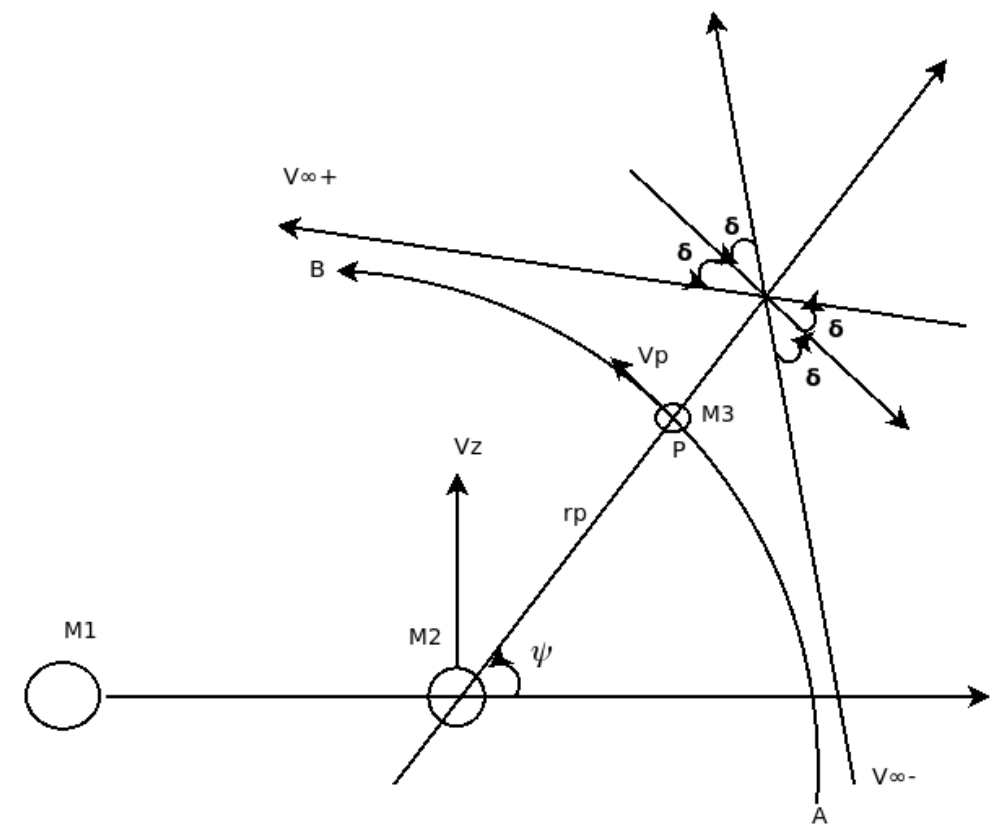

Figure 1. Gravitational maneuver due to the close encounter between two bodies. Based on Ferreira et al. ${ }^{22}$.

\subsection{Development}

For the input data for integration, the position and velocity components of the bodies, taken from the JPL Horizons website, were used. The mass of $(7,3290,009) 10^{10} \mathrm{~kg}$ and density of (119013) $\mathrm{kg} \mathrm{m}^{-3}$ were considered for the asteroid Bennu ${ }^{-26}$. These values can be found in Tables 1 and 2. We are not considering the composition and dimensions of the asteroid, as well as its rotation properties.

First, we found the closest approximation between the asteroid and Earth in a 100-year period from August 5, 2021, the date we began the study. All planets in the solar system, the Moon and the asteroid Bennu were used in our simulations.

This closest approach occurred on September 22, 2080, at a distance of approximately 0.005 au. From this date onwards, we collected the positions and velocities of all bodies and performed a simulation going 100 years back in time, reaching the new starting point. Then, we entered the values of the new starting point and proceeded to perform simulations over time applying velocity variations to the initial data of the asteroid Bennu. It is important to mention that, when performing the process of using the initial data values of the bodies extracted from the simulations in Mercury, going back in time, and returning the simulations forward again from the new data taken from the simulation, the bodies return in 2080 with the same orbital characteristics, being the same coordinates, velocities and relative distances. The variations of velocity start from $-50 \mathrm{~mm} / \mathrm{s}$ to $50 \mathrm{~mm} / \mathrm{s}$ with an increment of $10 \mathrm{~mm} / \mathrm{s}$. This methodology has already been used in many works found in the literature, such as Ahrens and Harris ${ }^{6}$, Carusi ${ }^{4}$, among others.

To better understand the phenomenon we are studying, we chose to divide the orbit of the asteroid into 36 parts, to collect more information about the application of impulses on the asteroid in different positions of its orbit and thus be able to more comprehensively assess each detail to which we might meet on a possible future mission. To carry out this process, the simulations were going back in time so that the impulses were applied at each position of the asteroid, with the difference that the positions are approximately $10^{\circ}$ in the mean anomaly of the orbit of the asteroid. Thus, the exposure period increases a little more than the initial period, which was 100 years, and the orbital period of the asteroid in question is approximately 1.2 years.

The impulses were applied at the center of mass of the asteroid, and in the direction of its orbital velocity vector. Variations in this impact angle will be developed in future.

\section{Results}

The simulations show us considerable variations in the closest approximation between the asteroid and the Earth for each impulse applied at each different position. These results can be found in Figures 2 and 3, where we separate the applications of negative variations in the velocity of the asteroid, when the impulses are applied opposite to the movement of the asteroid, in Figure 2, and for positive variations in the velocity of the asteroid, when the impulse was applied in the same direction of the movement of the asteroid, in Figure 3. These Figures show the closest approximation (in units of the radius of the Earth) 


\begin{tabular}{|l|l|l|l|}
\hline Bodies & $\mathrm{x}($ ua $)$ & $\mathrm{y}($ ua $)$ & $\mathrm{z}($ ua $)$ \\
\hline Mercury & $-2.896323130935082 E-01$ & $1.850577763255397 E-01$ & $4.169019903669655 E-02$ \\
\hline Venus & $-5.692473108639921 E-01$ & $-4.448853077740749 E-01$ & $2.674283485411453 E-02$ \\
\hline Earth & $6.855761418396654 E-01$ & $-7.477764241622177 E-01$ & $3.211115876042908 E-05$ \\
\hline Moon & $6.855266149744332 E-01$ & $-7.451016724453864 E-01$ & $1.230222158011698 E-04$ \\
\hline Mars & $-1.614905800650153 E+00$ & $3.955532232885259 E-01$ & $4.790302296698644 E-02$ \\
\hline Jupiter & $4.148571639394127 E+00$ & $-2.841339576414095 E+00$ & $-8.101578326784213 E-02$ \\
\hline Saturn & $6.390745065584204 E+00$ & $-7.624741172250260 E+00$ & $-1.217720468705376 E-01$ \\
\hline Uranus & $1.479576471988037 E+01$ & $1.307144787474826 E+01$ & $-1.431624026194872 E-01$ \\
\hline Neptune & $2.956816826343533 E+01$ & $-4.557431754532644 E+00$ & $-5.876364308100537 E-01$ \\
\hline Bennu & $-8.178122041042245 E-01$ & $6.126618626692276 E-01$ & $6.774426832917149 E-02$ \\
\hline
\end{tabular}

Table 1. Input values for the coordinates of each body as of August 5, 2021. Data taken from JPL NASA.

\begin{tabular}{|l|l|l|l|}
\hline Bodies & vx(ua/day) & vy(ua/day) & vz(ua/day) \\
\hline Mercury & $-2.090746004400052 E-02$ & $-2.253446087272209 E-02$ & $7.638025951147821 E-05$ \\
\hline Venus & $1.231235542811912 E-02$ & $-1.603266518952336 E-02$ & $-9.305216171721341 E-04$ \\
\hline Earth & $1.240809069382479 E-02$ & $1.156282606438427 E-02$ & $-1.150024366950303 E-06$ \\
\hline Moon & $1.184355116475734 E-02$ & $1.153246162528540 E-02$ & $4.458968850063871 E-05$ \\
\hline Mars & $-2.807629223103034 E-03$ & $-1.239693158153517 E-02$ & $-1.909313502062999 E-04$ \\
\hline Jupiter & $4.177326698222665 E-03$ & $6.587451407751826 E-03$ & $-1.208714010214003 E-04$ \\
\hline Saturn & $3.969863463512147 E-03$ & $3.576887186643701 E-03$ & $-2.197897896266317 E-04$ \\
\hline Uranus & $-2.628949090335184 E-03$ & $2.770739109958900 E-03$ & $4.429549807400130 E-05$ \\
\hline Neptune & $4.618822543692893 E-04$ & $3.128329541906231 E-03$ & $-7.516594581186683 E-05$ \\
\hline Bennu & $-1.309992480373342 E-02$ & $-1.191701206869430 E-02$ & $-1.210612917719958 E-03$ \\
\hline
\end{tabular}

Table 2. Input values for the velocity of each body as of August 5, 2021. Data taken from JPL NASA.

between the asteroid and Earth found in the simulation period on the vertical axis and the applied impulse position (mean anomaly in degrees) on the horizontal axis.

Looking at Figure 2, it is interesting and encouraging to note that, for impulses applied at some positions on the asteroid, the smaller variations in velocity were almost equivalent to the larger applied impulses. This is encouraging as it shows that we will not necessarily need a large impactor to deflect the asteroid, but rather determine a safe position for the intended objective in order for the asteroid to receive the boost. For example, applying the impulses at $25^{\circ}$ from the aphelion of the orbit of the asteroid (mean anomaly $155^{\circ}$ ), all impulses against the motion of the asteroid bring the asteroid even closer to the Earth. The opposite effect occurs when impulses are applied in the same direction of the movement of the asteroid, as we can see in Figure 3, where all the impulses move the asteroid away from the Earth. However, for impulses applied close to aphelion (mean anomaly $184^{\circ}$ ), both impulse scenarios moved the asteroid away from Earth, and this could be a safety region for the asteroid deflection scenario.

The same analysis can be performed by looking at the impulses applied at perihelion and its surroundings. Intending to follow the previous idea, analyzing Figure 2, we found that the impulse of $-40 \mathrm{~mm} / \mathrm{s}$ approached the asteroid in the mean anomaly of $334^{\circ}, 26^{\circ}$ before perihelion, while in the position closest to perihelion (mean anomaly of $4^{\circ}$ ), the impulse of -20 $\mathrm{mm} / \mathrm{s}$ approached the asteroid. The same happens for the impulses in the same direction of the motion of the asteroid, this can be observed in Figure 3, where, for the same positions, the largest impulses are $40 \mathrm{~mm} / \mathrm{s}$ and $50 \mathrm{~mm} / \mathrm{s}$, brought the asteroid even closer to the Earth. It is clear to us that the magnitude of the impulse in this region is crucial to whether the asteroid will come closer at some point or if it will move away from the Earth. It shows that, for a scenario where we would be forced to apply the impulse in this region, the magnitude of the impulse must be considered independent of whether we are going to apply the impulse contrary to the movement of the asteroid or in the same direction of it, and that, in a scenario of deflect the asteroid away from the Earth, larger impulses are not always the best alternatives.

An interesting result shown in Figure 2, would be for the impulse of $-10 \mathrm{~mm} / \mathrm{s}$ applied to the asteroid. We can see that, in just two situations, this impulse brought the asteroid even closer to the Earth (mean anomalies $165^{\circ}$ and $155^{\circ}$ ) and that, if we observe the impulse being applied between the anomalies of $324^{\circ}$ and $184^{\circ}$, it showed almost equivalent results to the largest impulses in the concept of moving the asteroid away and, in some positions, giving better results than larger impulses, for this objective. Looking at Figure 3, we found a similar scenario, changing only the positions where there is a closer approximation 


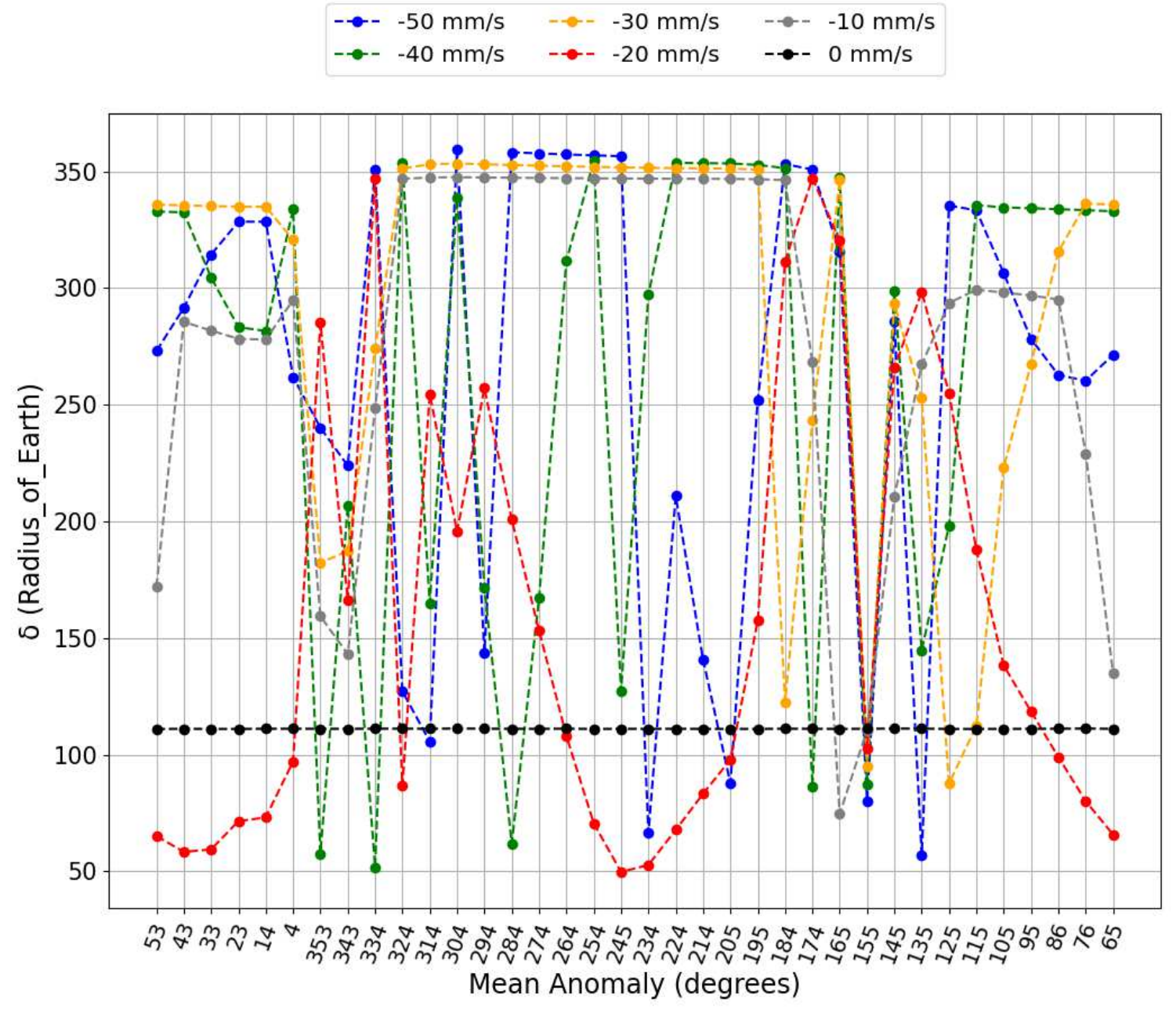

Figure 2. The closest approximations between the asteroid and the Earth along a period of about 100 years $(\delta)$ (y axis), after negative velocity variations at different mean anomalies ( $\mathrm{x}$ axis) to simulate different positions of the impulse application. The black dots are the closest approximations of the asteroid to the Earth without the application of impulse. The $\delta$ is in radius of Earth.

between the bodies due to impulses of $10 \mathrm{~mm} / \mathrm{s}$, where these impulses occur in anomalies of $343^{\circ}$ and $145^{\circ}$, and the positions of greater distances between bodies occur between mean anomalies in the interval from $353^{\circ}$ to $125^{\circ}$.

For impulses of $-20 \mathrm{~mm} / \mathrm{s}$, it is noticed that they present more scenarios where, possibly, the asteroid will approach even closer to the Earth instead of moving away. It is worth mentioning that, from the mean anomalies between $4^{\circ}$ to $86^{\circ}$, all the impulses of $-20 \mathrm{~mm} / \mathrm{s}$ brought the asteroid even closer to the Earth. This again occurs at the mean anomalies between $205^{\circ}$ and $264^{\circ}$. This fact caught our attention since, apparently, there would not be a specific reason why this could occur. Another interesting result is some observed jumps, such as the impulse of $-20 \mathrm{~mm} / \mathrm{s}$ applied at the $324^{\circ}$ and $334^{\circ}$ in the mean anomaly of the asteroid. It is notorious that, for impulses application at the position where the mean anomaly is $334^{\circ}$, the asteroid moves away from the Earth, while when the impulse is applied $10^{\circ}$ earlier, the asteroid will come even closer to the Earth. This result will be further detailed in the later sections. Looking at Figure 3, we found that, for the impulse of $20 \mathrm{~mm} / \mathrm{s}$, situations where the asteroid approached the Earth even closer than the other applied impulses are also presented. In these Figures, we notice that, for impulses of $-20 \mathrm{~mm} / \mathrm{s}$ and $20 \mathrm{~mm} / \mathrm{s}$, for each position, the results vary drastically, that is, a pattern is not maintained in relation to similar values in the greatest approximations in consecutive positions, except for Figure 3, where we see that, for the impulses applied in mean anomalies in the interval from $23^{\circ}$ to $43^{\circ}$, a brief pattern follows. As mentioned, we will address the $-20 \mathrm{~mm} / \mathrm{s}$ results a little more in order to better explain the results presented here.

Analyzing the results for the impulses of $-30 \mathrm{~mm} / \mathrm{s}$, we found a very similar scenario for the impulses of $-10 \mathrm{~mm} / \mathrm{s}$. We can see that the impulses of $-30 \mathrm{~mm} / \mathrm{s}$ brought the asteroid even closer to the Earth in just two situations; applying the impulse in the 


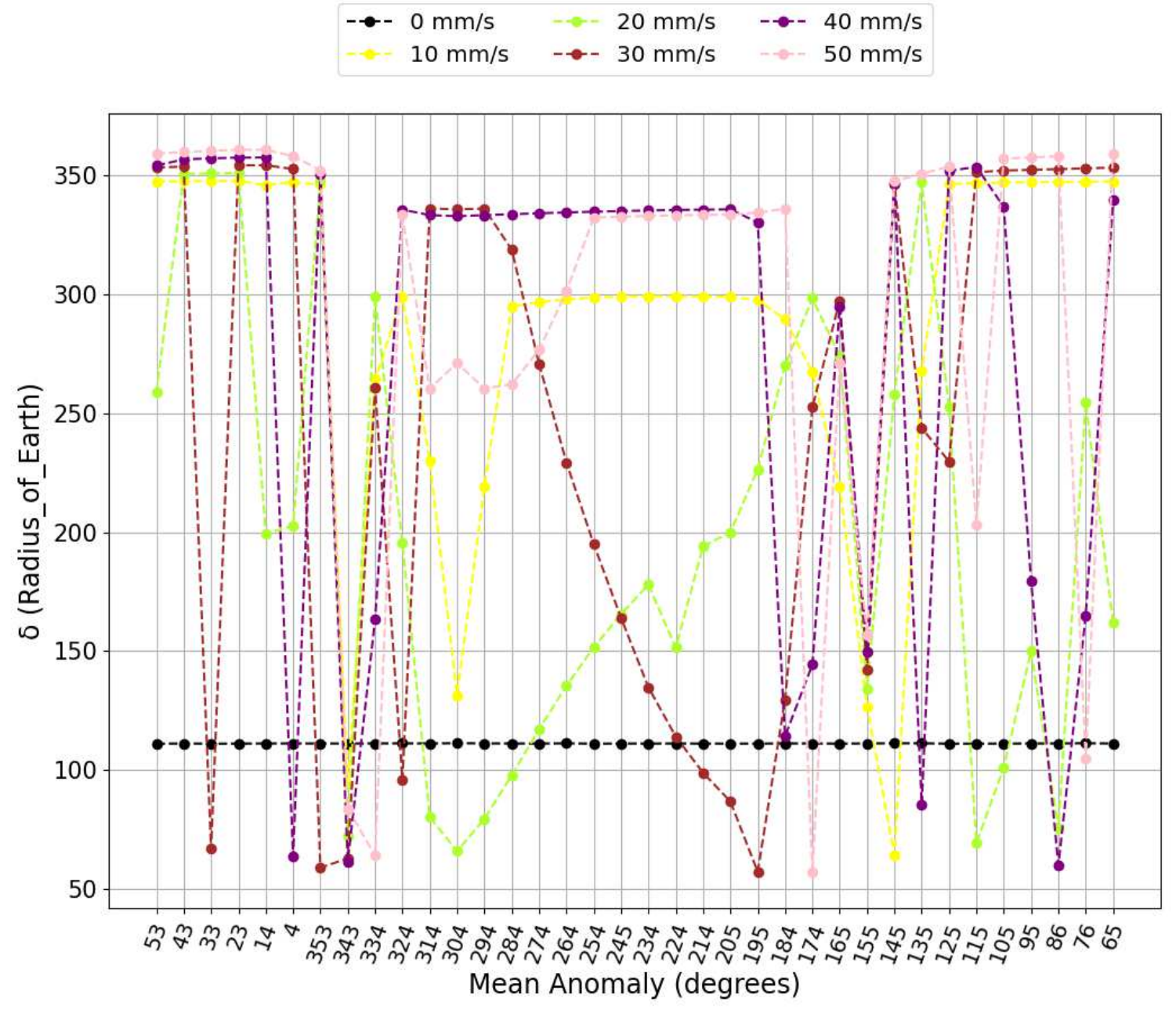

Figure 3. The closest approximations between the asteroid and the Earth along a period of about 100 years $(\delta)$ (y axis), after positive velocity variations at different mean anomalies ( $\mathrm{x}$ axis) to simulate different positions of the impulse application. The black dots are the closest approximation of the asteroid to the Earth without the application of impulse. The $\delta$ is in radius of Earth.

mean anomalies of $155^{\circ}$ and $125^{\circ}$, and, for our objective of moving the asteroid away from the Earth, it presented itself as an impulse very favorable to this purpose. It is still worth commenting that, even if this impulse presents good results, being even better than the impulse of $-10 \mathrm{~mm} / \mathrm{s}$, we are judging to be the most encouraging. We have to remember that we would triple the impulse, so it would require a larger impactor, which may not present a great advantage for its application, unless we are going to apply the impulse in the mean anomaly of $165^{\circ}$, which would also not be as necessary, since the impulse of $-20 \mathrm{~mm} / \mathrm{s}$ in this position would succeed. As for the impulse of $30 \mathrm{~mm} / \mathrm{s}$, this is one of the impulses that presented the most scenarios of bringing the asteroid closer to the Earth when the impulse is applied in the same direction of the movement of the asteroid. We will not, at this moment, talk about better or worse impulses, as we are arguing that the position where it occurs should be analyzed but, due to the practicality of a possible need for asteroid deflection, we believe that this impulse is not so encouraging.

Performing an analysis now on the largest impulses of $-40 \mathrm{~mm} / \mathrm{s}$ and $-50 \mathrm{~mm} / \mathrm{s}$, it is possible to observe good scenarios to move the asteroid away from the Earth, but we also noticed a great advantage in using smaller impulses, because, in many scenarios, they present results similar, or even move the asteroid away from the Earth while the larger impulses bring the asteroid even closer to the Earth. See the impulses applied in mean anomalies $135^{\circ}, 174^{\circ}, 205^{\circ}, 234^{\circ}, 284^{\circ}, 314^{\circ}, 334^{\circ}, 353^{\circ}$. Not taking into account the $155^{\circ}$ anomaly impulse, all other impulses brought the asteroid closer to the Earth. For the $40 \mathrm{~mm} / \mathrm{s}$ and $50 \mathrm{~mm} / \mathrm{s}$ impulses, shown in Figure 3, we found better results to move the asteroid away, but they are still at a disadvantage when compared to the smaller impulses.

We can see that for Figure 2, there are some positions where all the impulses moved the asteroid away from the Earth, like 
for the mean anomalies of $95^{\circ}, 105^{\circ}, 145^{\circ}, 184^{\circ}, 195^{\circ}, 274^{\circ}, 294^{\circ}, 304^{\circ}, 343^{\circ}$. We also find positions in Figure 3 where in the mean anomaly positions of $14^{\circ}, 23^{\circ}, 43^{\circ}, 53^{\circ}, 65^{\circ}, 95^{\circ}, 125^{\circ}, 155^{\circ}, 165^{\circ}, 184^{\circ}, 224^{\circ}, 234^{\circ}, 245^{\circ}, 254^{\circ}, 264^{\circ}$ and $274^{\circ}$, all impulses move the asteroid away from the Earth. These are positions that, for this case, would be good options to apply the impulse in order to move the asteroid Bennu away from the Earth.

As we can see, large variations occur in the closest approximations between the asteroid and the Earth for impulses applied outside the perihelion and aphelion position of the asteroid. Ferreira et al. ${ }^{22}$ showed that a satellite undergoing a propelled Swing By has the same characteristic as our results found here. Analyzing this work, we realize that we are working with the same idea, where in fact, we can employ the same explanation for the kinetic impact asteroid deflection scenario, where the impulses applied to the asteroid and the gravitational maneuver combine intensifying the variations in the orbit of the asteroid. We are just considering that this process occurs several times, generating an accumulation over time of the disturbances suffered by the asteroid and intensifying its energy variation.

Figures 2 and 3 give us interesting long-term ideas of the closest approximations between the bodies, and can serve as a guide to determine the best position to apply impulses in the orbit of a given asteroid. With that in mind, we can express them as Maps for Impulses in the Orbit of Asteroids (M.I.O.A.).

\subsection{Impulses in the perihelion and aphelion of the orbit of the asteroid.}

Looking at the M.I.O.A., we notice that, by dividing the orbit of the asteroid into 36 parts, we did not completely reach the positions of perihelion and aphelion, but positions close to them, with anomalies of $4^{\circ}$ and $184^{\circ}$ respectively. However, we find in the literature that the impulses applied in these positions must be considered ${ }^{14}$. Following this idea, we performed more simulations applying impulses in these two positions of the asteroid. The closest approximations between the asteroid and the Earth for each applied impulse is shown in Figure 4.

The black vertical line represents the value of the closest approximation of the asteroid to the Earth without impulses being applied. We found that for impulses applied at perihelion, only the impulse of $20 \mathrm{~mm} / \mathrm{s}$ brought the asteroid even closer to the Earth, while for the impulse applied at the aphelion of the asteroid, impulses of $-30 \mathrm{~mm} / \mathrm{s}, 10 \mathrm{~mm} / \mathrm{s}$ and $40 \mathrm{~mm} / \mathrm{s}$, bring the asteroid closer to the Earth, with the $10 \mathrm{~mm} / \mathrm{s}$ impulse at aphelion and the $20 \mathrm{~mm} / \mathrm{s}$ impulse at perihelion bringing the asteroid closer to the Earth by less than one mean Earth-Moon distance.

It is interesting to note that, for the smallest impulses, $-10 \mathrm{~mm} / \mathrm{s}$ and $10 \mathrm{~mm} / \mathrm{s}$, we achieved good results in terms of moving the asteroid away, as we have already seen in the previous subsection, with the exception already mentioned in the previous paragraph, the positive impulse (in the same direction as the motion of the asteroid), bringing the asteroid even closer to the Earth. We should also mention the impulse of $-20 \mathrm{~mm} / \mathrm{s}$, which for the mean anomaly of $4^{\circ}$ was bringing the asteroid even closer to the Earth, but, when applied at perihelion, it is capable of moving it away. That is, a small difference in the point of application of the impulse is capable of being decisive to bring the asteroid even closer or move it away from Earth.

Looking at Figure 4, we also found that, for the impulses applied at the perihelion of the orbit of the asteroid, all negative impulses moved the asteroid away from Earth. Something to analyze would be the region already mentioned for impulses of $-10 \mathrm{~mm} / \mathrm{s}$, that obtained the greatest results to move the asteroid away from Earth for this magnitude of impulse. We found that, by applying the $-10 \mathrm{~mm} / \mathrm{s}$ impulse in the aphelion of the orbit of the asteroid to approximately $36^{\circ}$ before the perihelion, the asteroid will be moving away with greater intensity. This system has a complex dynamics, therefore, it becomes difficult to make analytical predictions, which means that the only way to measure the effects is to perform numerical simulations, which is the way presented in this work, hence the importance and necessity of the present work. .

Figure 5 presents the approximations that occur between the asteroid and the Earth for the proposed simulation period, which is approximately 100 years. We limit the vertical axis, the approximations of the asteroid to the Earth $(\delta)$ in units of the radius of Earth, to 1000 radius of Earth to better visualize the approaches that occur over the period. We will have the impulses applied at perihelion in the upper figure and aphelion in the lower figure. These Figures will help us visualize what we have explained in relation to Figure 4.

We amplified the first two approximations within the mentioned limit and realized that, already in the first approximation between 19 years and 20 years after the applied impulses, it is already noticeable variations in the approximation, where we see that the intensity of the variations were proportional to the applied impulses. The second approximation makes the variations that occur even more clear, intensifying the variations after the first approximation, which makes us think that something else is intensifying these variations and not just the impulses applied in the orbit of the asteroid, since the variation is much larger than expected for a not so long time span. If we consider the next approximations we will see that the phenomenon is even more visible.

So, we realized that the various approximations of the asteroid with the closest bodies is interconnected with its natural trajectory and, after the applied impulses, these approximations generate variations in the perturbation, which will intensify with each new approximation mainly to the Earth due to its smaller relative distances between the asteroid and the planet than in relation to the other planets. In other words, what really generates a greater distance is the gravitational disturbance over the 


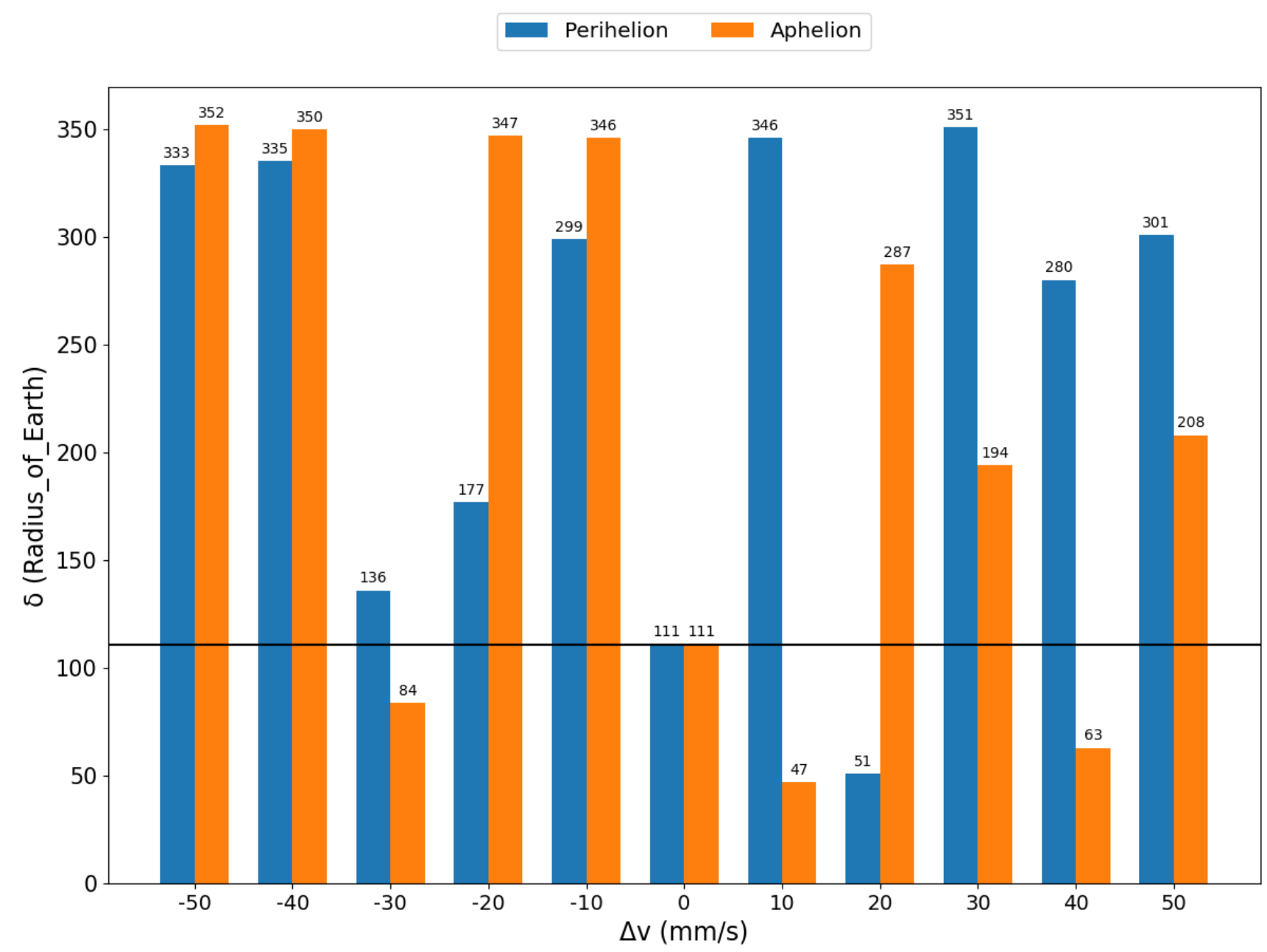

Figure 4. The closest approximations between the asteroid and the Earth $(\boldsymbol{\delta})$ (y axis), after velocity variations (x axis) for the impulse applied at perihelion (blue) and aphelion (orange). The horizontal black line indicates the value of the closest approximation of the asteroid to the Earth without the impulse. The exact value of the distance between the bodies is expressed above each bar.

years to which the asteroid will be exposed after the impulse. The impulse ends up being the trigger to change the behavior of the orbit of the asteroid, however, the factor that is really responsible for considerably altering the orbit of the asteroid are the variations that occur with each new close encounter between the asteroid and the Earth.

An equally important result is in relation to the date when the new largest approaches occur after the impulse. Looking at the upper Figure 5, we found that the asteroid will approach Earth even closer on September 23, 2054, for the impulse of 20 $\mathrm{mm} / \mathrm{s}$. For Figure 5, we have that the new dates where occur the closest approximations for the impulses of $-30 \mathrm{~mm} / \mathrm{s}, 10 \mathrm{~mm} / \mathrm{s}$ and $40 \mathrm{~mm} / \mathrm{s}$, as seen in Figure 4, are May 2, 2055, 1 of May 2080 and May 1, 2061, respectively.

\subsection{Impulses applied in the Mean Anomaly of $324^{\circ}$ and $334^{\circ}$.}

In the previous subsections, we mentioned some interesting regions where, when applying certain impulse values, the asteroid can move away or close in a range of approximately $10^{\circ}$ from the position of its application. This result is interesting, as it proves our idea of the importance of perfectly knowing the position of the impulse. We have already explained our interest in the impulse of $-20 \mathrm{~mm} / \mathrm{s}$, where the impulse showed the greatest number of scenarios where the asteroid approached Earth even closer and also the existence of a jump between the impulses applied at the mean anomalies of $324^{\circ}$ and $334^{\circ}$. This difference found in the two positions is the target of this section, since we found intriguing the fact that in one position the asteroid is even closer to the Earth and, in the next position, there is a great distance of the asteroid. We will dedicate this section exclusively to the impulse of $-20 \mathrm{~mm} / \mathrm{s}$ applied in these two positions. 


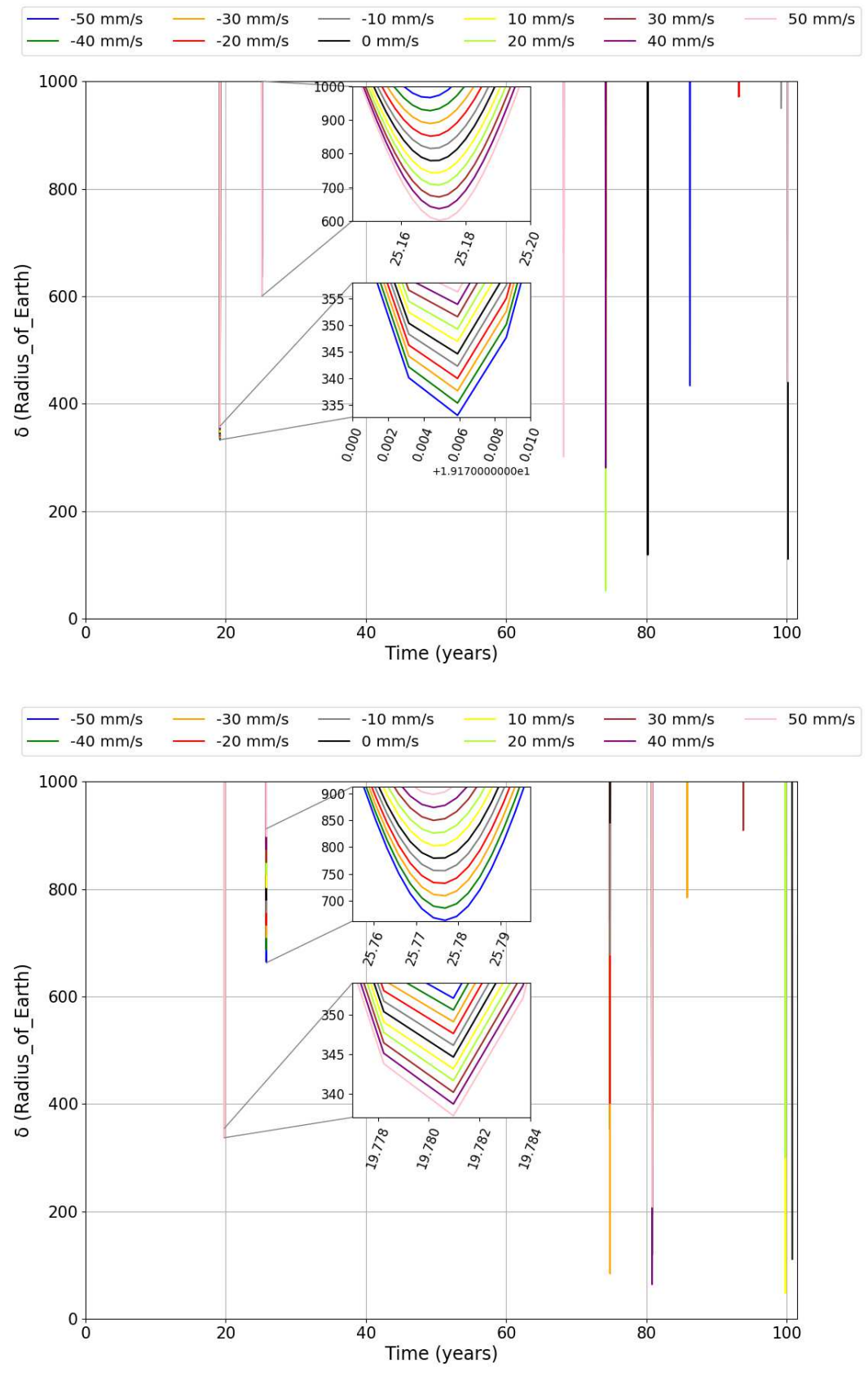

Figure 5. The relative distances between the asteroid and the Earth $(\delta)$ for the impulse applied at the point of perihelion (Top) and aphelion (Upper). We zoomed in on the first two close encounters to better visualize the variations in the approach between the asteroid and Earth.

We have already explained that the asteroid will have several close encounters with the Earth over the years, and that, with each close encounter it suffers gravitational perturbation from the planet. When we apply an impulse, we then notice that it has a variation in its relative distance $(\delta)$ to the planet in the first 20 years of simulation. This variation in the relative distance between the asteroid and the Earth is intensifying with the several close encounters and we realize that each impulse applied has its own characteristic.

Figure 6 shows the behavior of the relative distances between the asteroid and the Earth $(\delta)$ for a period of approximately 100 years, for the impulse of $-20 \mathrm{~mm} / \mathrm{s}$ applied in the mean anomalies of $324^{\circ}$ and $334^{\circ}$. In Figure 5 we have enlarged the region surrounding the first two close encounters between the asteroid and the Earth, remembering that we are considering the 


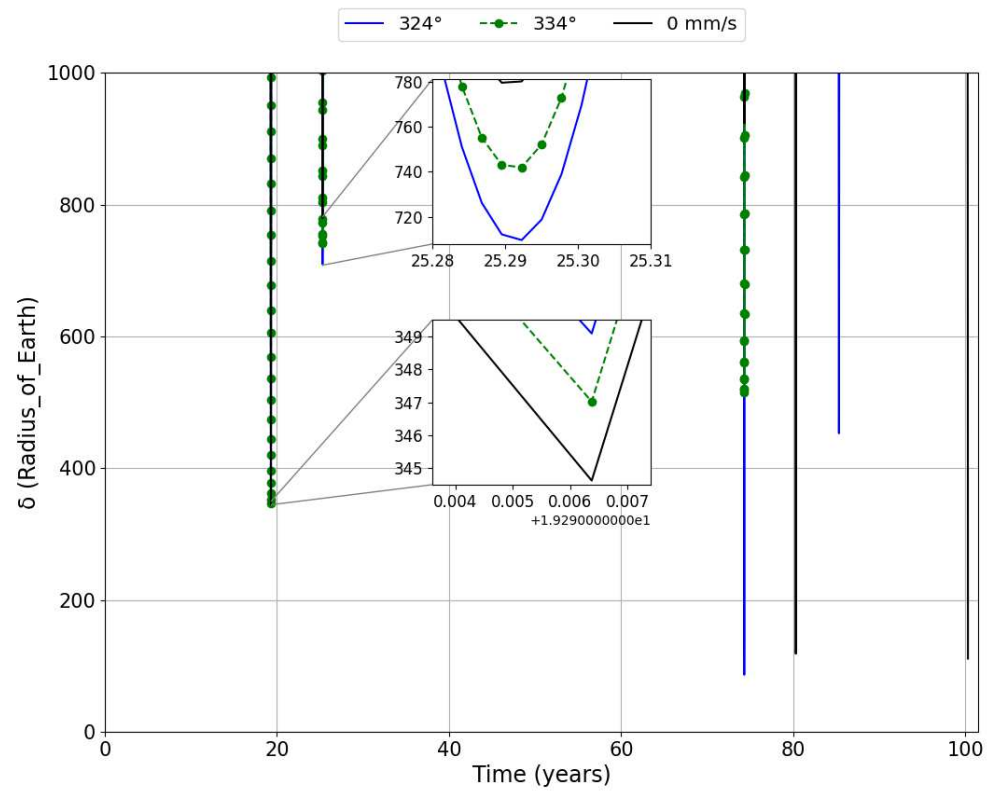

Figure 6. The relative distances between the asteroid and the Earth $(\delta)$ for the impulse applied at the mean anomalies of $324^{\circ}$ (blue line) and $334^{\circ}$ (green dashed line). We zoomed in on the first two close encounters to better visualize the variations in the approach between the asteroid and the Earth.

region in the Figure as the first two encounters within the range of 0 to 1000 radius of the Earth of the relative distance between the bodies. Other encounters are occurring before those presented, but at a relatively large distance. Analyzing the Figures, although we have made this truncation in the Relative Distance Figures, and these close encounters between the asteroid and the Earth do not appear there because they are at greater distances, they should not be neglected in relation to the effect they produce, since we realized that the first close encounter that we are expanding already shows a significant variation in the relative distance between asteroid and Earth if we take into account the impulses applied.

We can see from the very first large encounter that the asteroid is offset by approximately 5 radius of Earth and 2 radius of Earth for the impulse applied at the mean anomalies of $324^{\circ}$ and $334^{\circ}$, respectively. At the next large encounter, we find that the asteroid is brought closer to the Earth at approximately 55 radius of Earth for the impulse applied in $324^{\circ}$, and approximately 40 radius of Earth for the impulse applied in $334^{\circ}$. After analyzing these regions, we can now look at the Figure as a whole. We see that when the impulse is applied on the mean anomaly of $324^{\circ}$, it brings the asteroid even closer to the Earth between 70 and 80 years after its application. On December 16, 2054 it is at a distance of approximately 87 radius of Earth, in addition to the Earth-Moon distance.

Our objective here is to study the methods to move the asteroid away from Earth. However, analyzing this closer approximation of the asteroid to the Earth in this period, it is noteworthy that the approximation did not generate an impact with the Earth, but the asteroid was moved away from the Earth at a considerably safe distance, that is, even with the close approximation in 2054 becoming the shortest relative distance between the asteroid and Earth after the impulse in the period studied here, the results show that this close approximation can contribute to a largest deflection afterwards. Therefore, we can not rule out the idea that, for a long-term situation, we could bring the asteroid even closer, but in a safe region, and then, through gravitational perturbation, move it away completely in the dangerous period. As for the application of the impulse in the mean anomaly of $334^{\circ}$, we realized that the closest approximation of the asteroid to the Earth becomes the first close encounter with the Earth in approximately 19 years, on December 3, 1999, more precisely, at a distance relative of approximately 347 radius of Earth.

Figure 7 shows the behavior of the semi-major axis of the asteroid in relation to time. We found that, when going through the first close encounter, the asteroid naturally undergoes a first gravitational perturbation. This perturbation decreases the semi-major axis of the asteroid until it enters an 'almost neutral' region, where we can see that the asteroid remains for a safe period. At this moment the asteroid is making its move away from the perigee with the Earth. In the second close encounter, where we find a greater variation in the relative distance between the asteroid and the Earth, it is observed that the semi-major axis decreases and then decreases again, but to a smaller magnitude. We enlarged this region to better study the phenomenon, 


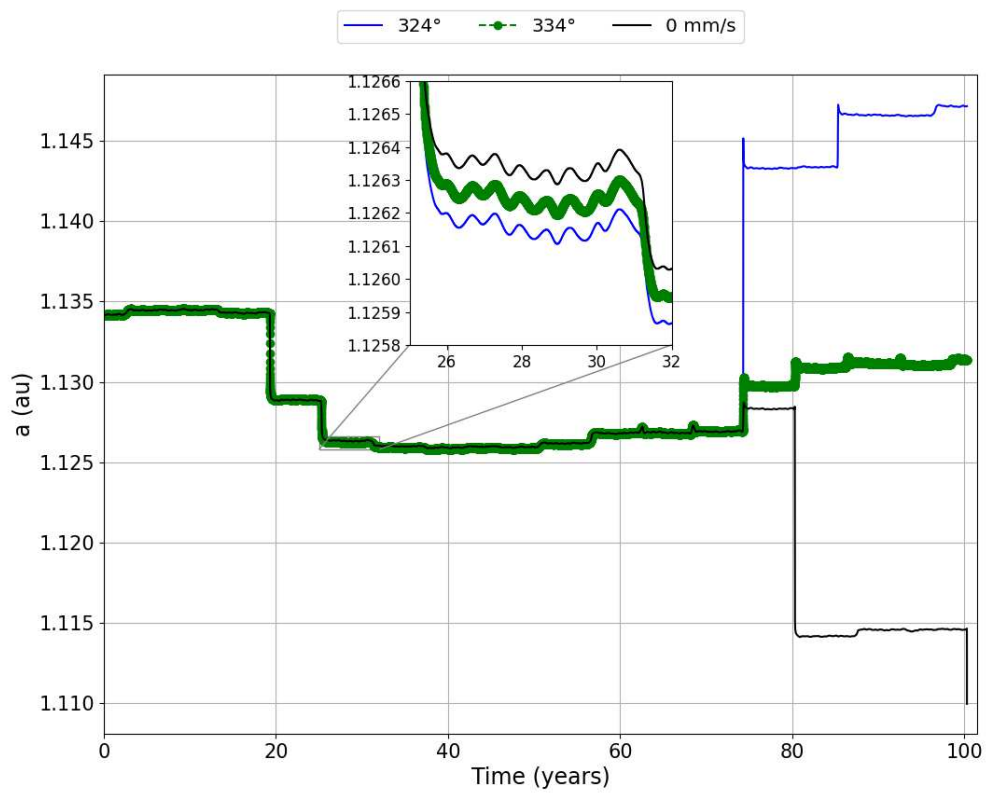

Figure 7. Behavior of the semi-major axis with respect to time for the impulse of $-20 \mathrm{~mm} / \mathrm{s}$ applied at the mean anomalies of $324^{\circ}$ (blue line) and $334^{\circ}$ (green line). We zoomed in on the region corresponding to the second largest close encounter to better visualize the semi-major axis variations of the asteroid since, at this time, the asteroid has significant variations in its relative distance from the Earth.

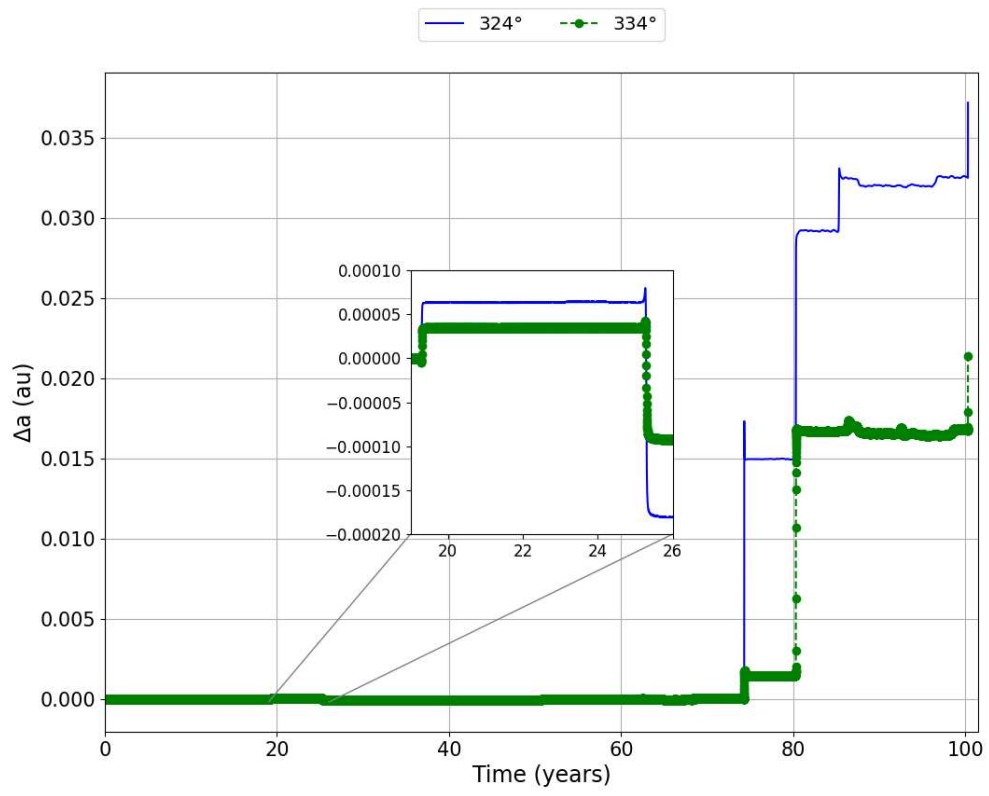

Figure 8. Difference of the semi major axis in relation to time $(\Delta \mathrm{a} / \Delta \mathrm{t})$ for the impulse applied at the mean anomalies of $324^{\circ}$ (blue line) and $334^{\circ}$ (green line). We zoomed in on the region corresponding to the second largest close encounter to better visualize the variations of the semi-major axis difference of the asteroid.

as we can see the impulses decrease the semi-major axis with more intensity than the natural phenomenon of close encounters without impulse application. We can see this variation better in Figure 8, where we show the difference $(\Delta \mathrm{a})$ of the semi-major axis with the impulses in relation to the semi-major axis without the impulse. 


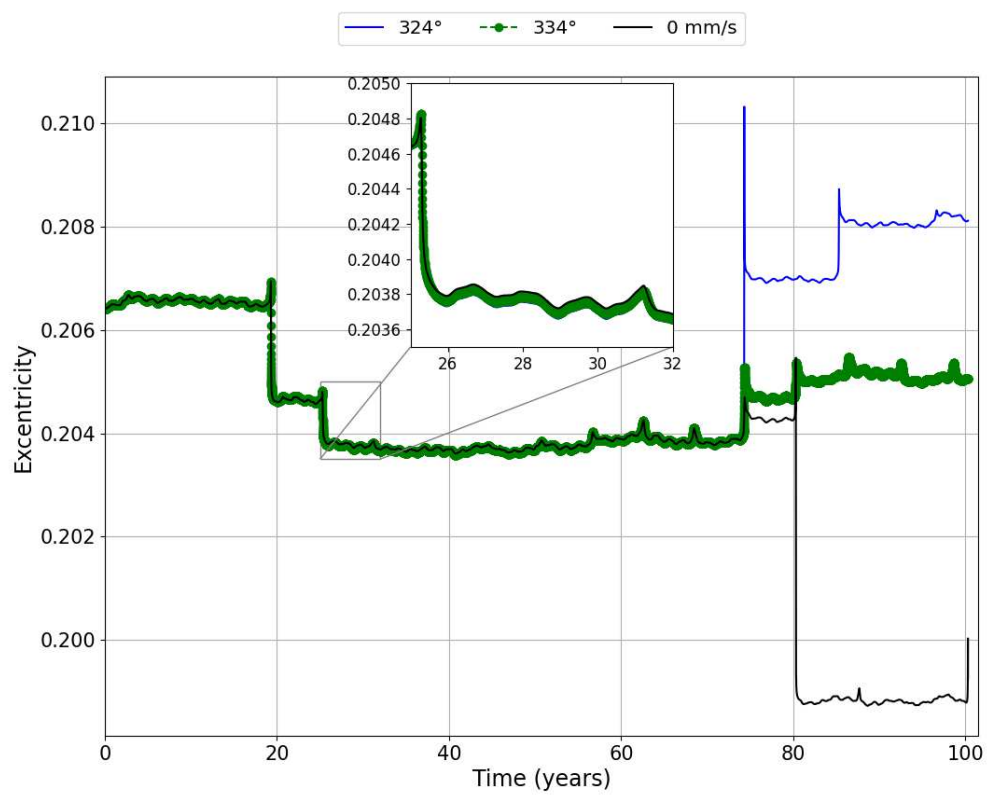

Figure 9. Behavior of asteroid eccentricity (e) over time for the impulse of $-20 \mathrm{~mm} / \mathrm{s}$ applied at the mean anomalies of $324^{\circ}$ (blue line) and $334^{\circ}$ (green line). We zoomed in on the region corresponding to the second largest close encounter to better visualize the variations in the asteroid's eccentricity, since at this time the asteroid has significant variations in its relative distance from the Earth.

Remembering expression 2, where we can associate the Energy of the asteroid with its semi-major axis, we will notice that, by decreasing the semi-major axis even more, the asteroid loses energy, as it is becoming more and more negative. This variation in energy will cause the asteroid to change its position relative to the Earth. Looking at the close approximation of the asteroid to the Earth in 2054 provided by the impulse applied at $324^{\circ}$, we will see that large semi-major axis differences occur, where the semi-major axis is increasing considerably, causing the asteroid to gain energy. The same happens for the impulse applied at $334^{\circ}$, however we will see that the semi-major axis increase occurs after 80 years after the impulse, causing the semi-major axis to remain value close to the one just after the passage before. In other words, the semi-major axis does not decrease drastically as it naturally does without impulse, nor does it increase as much as in the situation where the impulse is applied at $324^{\circ}$.

Figures 9 and 10 show the evolution of the eccentricity and the eccentricity differences when are applied impulses. We found variations for the eccentricity in the same moments as the variations for the semi-major axis. We noticed that, in the second close encounter of the asteroid with the Earth, the eccentricity decreases, which makes the velocity differences decrease due to the change in the distance between perihelion and aphelion. However, these variations are very small, in the order of $10^{-5}$. A very different scenario can be found in 2054 where, for the impulse applied at the mean anomaly of $324^{\circ}$, there is a large increase in the eccentricity of the asteroid, which leads to an increase in the velocity difference, since we will have a closer perihelion and the aphelion will be further away. At the next close encounter we find yet another big increase in eccentricity, but for the two impulse-applying positions.

These variations in asteroid velocity differences are crucial to understanding our results, since, by changing the velocity of the asteroid, we were able to increase its velocity to the point where asteroid was moved forward when passing by the position that would be the close encounter with the Earth naturally. It approach Earth, if the Earth naturally passes by the rendezvous region first than the asteroid, or delays the asteroid to the point where it misses the close rendezvous with the Earth, so the Earth passes through the rendezvous point earlier than the asteroid. If the asteroid naturally passes the nearby rendezvous point earlier than the Earth, it will pass closer to the Earth due to this delay. We can see this scenario better in Figure 11.

In the top of Figure 11 we can see the positions of the asteroid in relation to the Earth in a period of 100 days, considering in this time interval the positions before and after the second close encounter. We also indicate the movement of the Sun in relation to the Earth to better visualize the movement that the asteroid performs due to the gravitational attraction by the Sun. It is already visible in this figure the variations in the approximations that occur between the asteroid and the Earth after the impulses are applied, where we see that the impulse applied to the mean anomaly of $324^{\circ}$ brings the asteroid a little closer to 


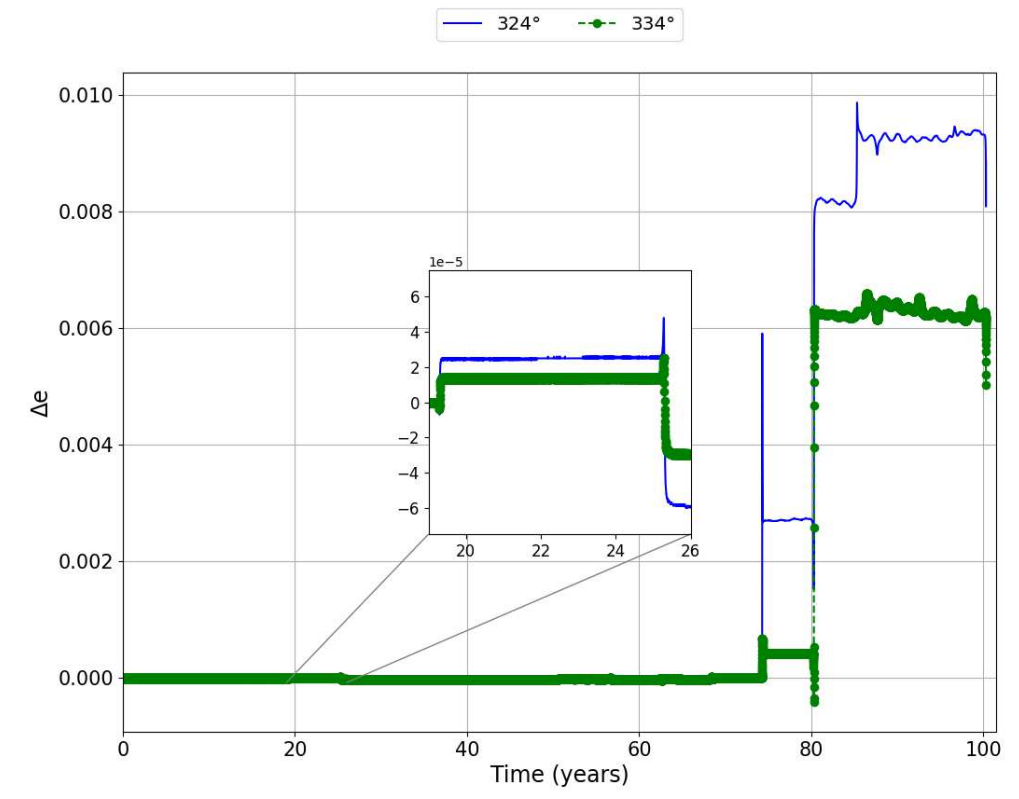

Figure 10. Difference between eccentricity with impulse applied and without impulse applied over time $(\Delta \mathrm{e} / \Delta \mathrm{t})$ for the impulse applied at the mean anomalies of $324^{\circ}$ (blue line) and $334^{\circ}$ (green line). We zoomed in on the region corresponding to the second largest close encounter to better visualize the variations in the eccentricity difference of the asteroid.

the planet. It is also natural to think of this close encounter between the bodies as a 'Swing By' between the asteroid and the Earth, where it will vary its energy due to the gravitational maneuver. At this moment we also notice that the asteroid is a little behind in relation to its natural movement. We can detect this fact in the last position of the asteroid in both Figure 11 (P5), which was explained by the analysis of the semi-major axis and eccentricity evolutions.

Analyzing now the bottom of Figure 11, we found even more visible the gravitational maneuver occurring due to the greater proximity between the bodies. It is easy at this point to see that the asteroid is far ahead of its natural motion at this position, even at the start of the position of the asteroid when the impulse is applied at the mean anomaly of $324^{\circ}$. We noticed that the asteroid already started its position in the time interval (P1) closer to the Earth when compared to its motion without impulse. When the impulse is applied at $324^{\circ}$, the point P1 is very near $\mathrm{P} 2$ of the motion without impulse, which we see on a smaller scale for the trajectory corresponding to an impulse applied at $334^{\circ}$. It is also very clear in Figure XX. This is even more evident in the last position of the asteroid (P5), when we see that the asteroid is much further ahead than its natural position.

This figure proves our whole idea that, over time, the asteroid undergoes several 'Swing By' with the Earth, changing its energy expressed by equation 3 . These variations change the velocity of the asteroid enough to delay its passage by the closer encounter to the Earth, causing it to either move away or even closer to the Earth. The same condition applies to the case where the asteroid advances its passage by the close rendezvous point with the Earth due to its velocity increase. This is an idea that was already initially addressed by Carusi et al. ${ }^{4}$, Park and Ross ${ }^{14}$, Ledkov et al. ${ }^{5}$, among others. We are now applying this idea for long periods and mapping the best positions to apply impulses by kinetic impact through the M.I.O.A. (Figures 2 and 3) and Figure 4, in addition to trying to clearly explain the 'Swing by' phenomenon within the asteroid diversion scenario.

\section{Discussion}

The results presented here show that the Swing By becomes important in the asteroid deflection technique over a relatively long period, where the position of application of the impulse must be considered in the approach. Knowing that for larger impulses we also need a larger-mass impactor, and for the feasibility of using this deflection method, our results show that due to the several close encounters of the asteroid with the Earth, we do not really need a large impulse to move the asteroid away, but we need to choose the best positions to apply the impulses. This result has already been mentioned in other works, such as Carusi ${ }^{4}$.

We observed that the impulses applied against the movement of the asteroid, applied in the perihelion of its orbit, have the ability to move the asteroid away from the Earth. The impulse of $10 \mathrm{~mm} / \mathrm{s}$ showed to be one of the most effective when applying impulses in the perihelion of the orbit for the case studied here. However, some applications of impulse in the aphelion of the orbit of the asteroid also showed good results, such as impulses of $-10 \mathrm{~mm} / \mathrm{s}$ and $-20 \mathrm{~mm} / \mathrm{s}$, which are in the sense of using 

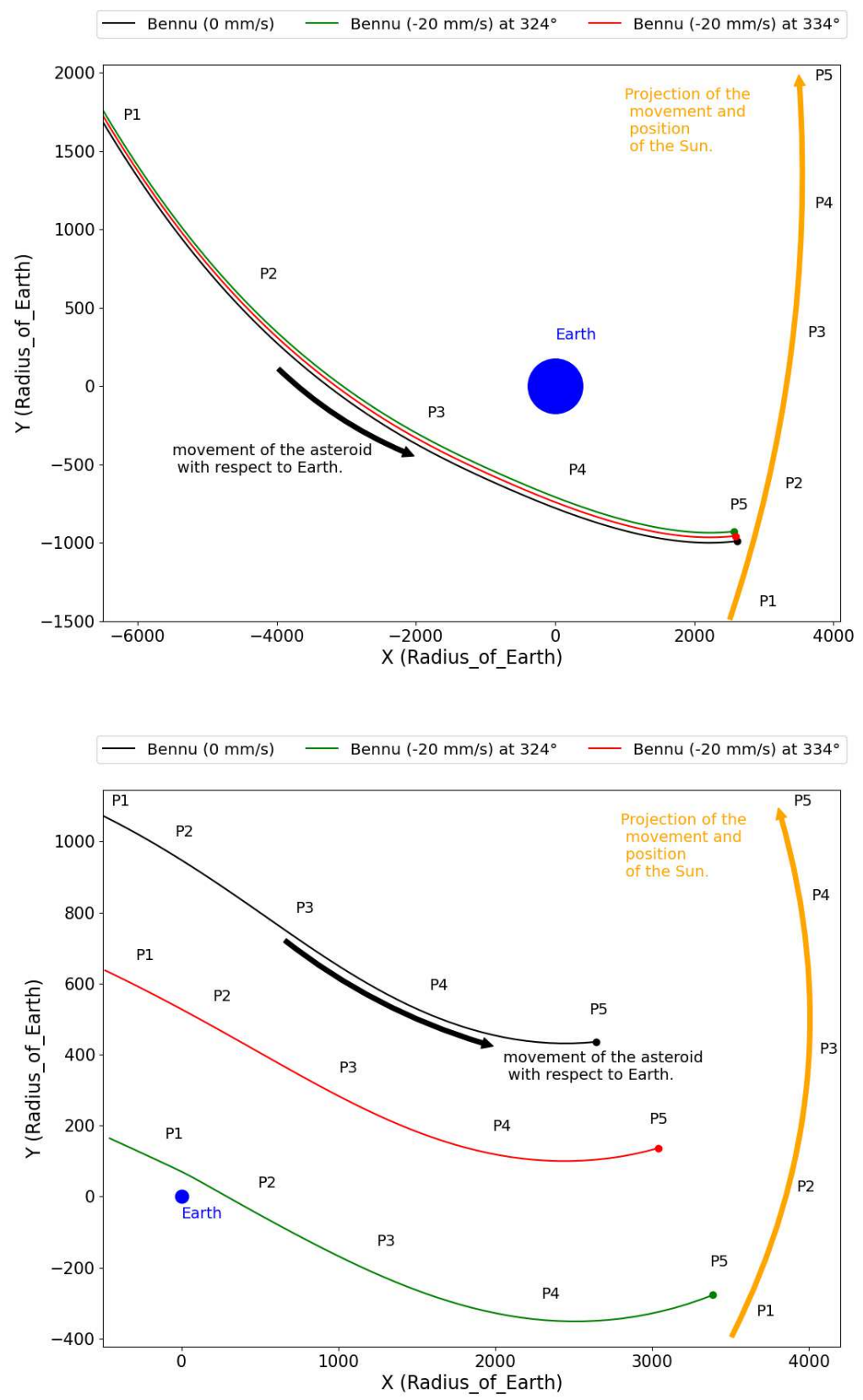

Figure 11. The position of the asteroid relative to the Earth without impulse and with an impulse of $-20 \mathrm{~mm} / \mathrm{s}$ applied at the mean anomalies $324^{\circ}$ (green line) and $334^{\circ}$ (red line), for the second largest encounter with Earth in approximately 25 years after the boost (top) and for the largest close encounter between the asteroid and the Earth in 2054, between 70 and 80 years after the push (bottom). We call P1, P2, P3, P4 and P5 the relative positions between the asteroid and the Sun, that is shown with respect to the Earth (blue).

smaller impactors. We also show, using the M.I.O.A., that we have some other good possibilities along the orbit of the asteroid to cause the impulse. For example, if we apply negative impulses to the velocity of the asteroid, for a scenario where we want to move it away in larger magnitude, we could apply the smallest impulse studied here among the regions between aphelion of the orbit of the asteroid and the position $36^{\circ}$ before the perihelion of the orbit of the asteroid. As for positive impulses, we have that this region starts a little before the perihelion and goes up to $55^{\circ}$ before aphelion, at the mean anomaly of $135^{\circ}$. We take this result as very encouraging, since we present good results that can be used according to the best strategies regarding the 
planning of possible missions, since we will be able to identify the best position to apply the impulse and also analyze the best scenario, taking into account economics factors. Like, for example, looking for a scenario where the asteroid is not too far from the Earth to apply the impulse, generating economy in the mission. We will not go into detail here, but it is something relevant.

The main point of our work remains to show that, using several close encounters between the asteroid and the Earth, it performs a natural Swing By with the Earth, and when we apply impulses in its orbit, the asteroid suffers variations in its relative distance with the Earth that generate variations in its energy, so the asteroid changes its velocity can both delay or advance a passage that would occur with the Earth in a period of approximately 100 years. This factor is responsible for bringing it closer, or away from the Earth.

\section{References}

1. Chourey, K. D. R. M. . S. C., S. Determining the momentum transfer in regolith-like targets using the tum/lrt electro-thermal accelerator. Planet. Space Sci. 194, 105112 (2020).

2. Park, . M. D. D., S. Y. Mission functionality for deflecting earth-crossing asteroids/comets. J. Guid. Control. Dyn. 26(5), 734-742 (2003).

3. Broschart, S. B. \& Scheeres, D. J. Control of hovering spacecraft near small bodies: application to asteroid 25143 itokawa. J. Guid. Control. Dyn. 28(2), 343-354. (2005).

4. Carusi, V. G. B. D. G. . B. A., A. Deflecting neos in route of collision with the earth. Icarus 159(2), 417-422 (2002).

5. Ledkov, e. a., Anton. Small near earth asteroids and gravity assist maneuvers as basic constituents of planetary defense against hazardous sky objects. SpaceOps 2014 Conf. (2014).

6. Ahrens, T. J. \& Harris, A. W. Deflection and fragmentation of near-earth asteroids. Nature 360(6403), 429-433 (1992).

7. Holsapple, H. K., K.A. Momentum transfer in asteroid impacts. i. theoryandscaling. Icarus 221, 875-887 (2016).

8. Housen, K. R. \& Holsapple., K. A. On the fragmentation of asteroids and planetary satellites. Icarus 84.1, 226-253 (1990).

9. Sanchez, V. M. . R. G., J. P. Consequences of asteroid fragmentation during impact hazard mitigation. J. Guid. Control. Dyn. 33(1), 126-146 (2010).

10. Hirabayashi, D. A. B. F. E. G. R. D. C. M. P. C. A. F. . N. S. P., M. Assessing possible mutual orbit period change by shape deformation of didymos after a kinetic impact in the nasa-led double asteroid redirection test. Adv. space research 63(8), 2515-2534 (2019).

11. Durda, W. J. D. C. S. H. K. R. G. D. J. M. S. . S. M. M., D. D. Laboratory impact experiments with decimeter-to meter-scale targets to measure momentum enhancement. Planet. Space Sci. 178, 104694 (2019).

12. Cheng, A. J. K. B. R. A. S. S. A. R. C. . U. S., A. F. Asteroid impact and deflection assessment mission. Acta Astronaut. 115, 793-799 (2015).

13. Cheng, A. F. e. a. Asteroid impact \& deflection assessment mission: Kinetic impactor.aida team. Avances Space Res. 121, 27-35 (2016).

14. Park, . R. I. M., S. Y. Two-body optimization for deflecting earth-crossing asteroids. J. Guid. Control. Dyn. 22(3), 415-420 (1999).

15. Conway, B. A. Near-optimal deflection of earth-approaching asteroids. Planet. Space Sci. 24(5), 1035-1037 (2001).

16. Izzo, D. On the deflection of potentially hazardous objects. In 15th AAS/AIAA Space Flight Mech. Conf. (2005).

17. Izzo, D. Optimization of interplanetary trajectories for impulsive and continuous asteroid deflection. J. guidance, control, dynamics 30(2), 401-408 (2007).

18. Negri, S. B. . A. F. B. A., R. B. Mapping trajectories of an asteroid that is deflected by a collision. (2018).

19. Chambers, J. E. A hybrid symplectic integrator that permits close encounters between massive bodies. Mon. Notices Royal Astron. Soc. 304.4, 793-799 (1999).

20. Danby, J. Fundamentals of celestial mechanics. fcm (1992).

21. Prado, A. Powered swing-by. J. Guid. Control. Dyn. (1996).

22. Ferreira, P. A. F. . W. O. C., A. F. A numerical mapping of energy gains in a powered swing-by maneuver. Nonlinear Dyn. 89(2), 791-818 (2017).

23. Broucke, R. The celestial mechanics of gravity assist. In Astrodyn. Conf. 4220 (1988). 
24. Kovácová, e. a., M. 101955 bennu and 162173 ryugu: Dynamical modelling of ejected particles to the earth. Planet. Space Sci. 185, 104897 (2020).

25. Lauretta, D. D. N. B. C. A. e. a., D. S. 101955 bennu and 162173 ryugu: Dynamical modelling of ejected particles to the earth. Planet. Space Sci. 185, 104897 (2019a).

26. Scheeres, M. J. W. F. A. S. e. a., D. J. The dynamic geophysical environment of (101955) bennu based on osiris-rex measurements. Nat. Astron. 3, 352-361 (2019).

\section{Acknowledgements (not compulsory)}

The publication has been prepared with the support of the RUDN University Strategic Academic Leadership Program.

We would like to thank FAPESP proc. 2016/024561-0, 2018/17864-1 and CNPq proc 305210/2018-1, 309089/2021-2 for funding and contributing to this work.

\section{Author contributions statement}

Chagas,B.S wrote the main version of the manuscript, generate data and prepared the figures. Chagas,B.S, A.F.B.A. Prado and O. C. Winter contributed on its conception, analysis, interpretation of data and revisions.

\section{Competing interests}

The authors declare that they have no conflicts of interest with research institutions, professionals, researchers, and/or financial supports.

\section{Data availability}

All data generated or analyzed during this study are included in this published article in form of figures.For more information, contact Bruno Chagas Santos (bruno.ba.987@gmail.com or bruno.chagas@unesp.br).

\section{Ethical approval}

The submitted work is original and not have been published elsewhere in any form or language. The work presents the results of a single study. The results are presented clearly, honestly, and without fabrication, falsification, or inappropriate data manipulation. No data, text, or theories by others are presented as if they were the author's own.

\section{Additional information}

Correspondence and requests for materials should be addressed to B.C.S. 